\title{
Le COMPleXe de la figure Patriarcale dans le Passe SIMPLE DE DRISS CHRAÏBI : LE TIRAILLEMENT ENTRE MODERNISME ET HERITAGE CULTUREL. Maï Abdelghafar Farid
}

\author{
Mai_Abdelghafar@lan.kfs.edu.eg
}

Maître de conférences, Faculté des langues, Département de la langue française, Université de Kafrelsheikh.

Toute création littéraire prend origine de sa portée par rapport à son environnement littéraire et idéologique. Elle opère principalement à l'intérieur d'un environnement culturel qui lui sert à la fois de cadre de référence et d'appréciation. Le lecteur occupe également un rôle primordial dans le mouvement de la réception d'une œuvre littéraire, c'est grâce à ces éléments dans le domaine de la littérature que l'œuvre acquiert tout son sens et son statut.

L'appartenance d'une œuvre à un champ littéraire en particulier est un accommodement entre l'espace environnemental et l'imaginaire de l'auteur. Cette appartenance figure principalement chez les auteurs maghrébins d'expression française dans l'adhésion à un groupe ou à une «tribu », mais était également la conséquence directe de la colonisation française et de sa présence en Afrique du nord, colonisation en Algérie, protectorat en Tunisie et au Maroc.

La littérature maghrébine d'expression française s'est donc traduite au Maghreb après l'indépendance de ces trois pays. Avant tout mise en place par des Français installés au Maghreb, elle devint par la suite l'apanage des Algériens, des Marocains et des Tunisiens.

Ce type de littérature est apparu à la suite des mouvances de la réalité culturelle des pays du Maghreb. Elle exprimait d'abord l'attachement à la terre natale des français de la deuxième et troisième génération - pour prendre l'exemple de l'Algérie française vivants sur le sol du pays colonisé, tel que Camus, Roblès etc. 
Orientée principalement contre le pays colonisateur avant l'indépendance, une littérature anticoloniale a ainsi émergé, créée par des écrivains maghrébins aspirant à une identité formellement maghrébine. Elle fut toujours écrite pour des lecteurs de 1'Hexagone. Elle eut pour but de persuader ce public de la légitimité de la bataille et de l'engagement littéraire au service de l'indépendance. Cette littérature anticoloniale se manifeste aussi au Maroc et plus sereinement en Tunisie. "En scrutant de près l'œuvre de l'indigène nord-africain, on constate l'omniprésence d'un " personnage » très important, souvent déguisé mais toujours là : le colonialisme »1.

Aujourd'hui on peut parler d'une littérature d'expression française à part entière dans les pays du Maghreb. Bien que cette littérature soit d'influence coloniale, on retrouve néanmoins des caractéristiques différentes dans chacune des littératures de ces trois pays «...Si on a longtemps parlé de littérature maghrébine au singulier, le pluriel s'impose aujourd'hui en raison des spécificités sociopolitiques et des particularités culturelles de chaque pays... $»^{2}$.

Ce mouvement compte des grands auteurs de littérature de renommée mondiale tels que Driss Chraïbi et Tahar Ben Jalloun, Tahar Bekri, Assia Djebar, Mohamed Dib, et d'autres. Ces auteurs sont eux-mêmes l'expression d'une identité perdue suite aux séquelles du colonialisme, une identité attachée autrefois à la notion de vie en groupe, en tribu, mais désormais influencée par la liberté aspirée par les espoirs d'une émancipation à l'occidentale. «Ces écrivains maghrébins dévoilent des aspects d'euxmêmes et de leurs sociétés, de leurs problèmes d'identité, de leurs conflits avec leurs compatriotes et avec des autres. Ils ont donné autrefois à voir une image d'eux-mêmes différente de celle proposée par la littérature des Français » ${ }^{3}$.

\footnotetext{
${ }^{1}$ Isaac Yetiv, L'aliénation dans le roman maghrébin contemporain, in ; Revue des mondes musulmans et de la Méditerranée, 1974, nº18, p. 152.

${ }^{2}$ ARON, Paul, SAINT-JACQUES, Denis, VIALA, Alain, le dictionnaire de littérature, Paris, PUF, 2002, p.434.

${ }^{3}$ Jean Déjeux, L'étrangère dans la littérature maghrébine de langue française, in; Hommes \& Migrations, 1993, n 1167, p. 36.
} 
On assiste par conséquent, chez ces auteurs à une sorte de paratopie ${ }^{4}$ qui caractérise ainsi à la fois la condition « de littérature et celle de tout créateur $»^{5}$. En conséquence l'auteur-romancier jongle de la frontière mouvante entre la réalité de la société, d'une géographie et d'un espace littéraire paratopique. « En fait l'Art n'a pas d'autre lieu que ce mouvement, l'impossibilité de se fermer sur soi et de se laisser absorber par cet autre qu'il faut rejeter mais dont on attend la reconnaissance ${ }^{6}$.

Il s'agit bien du cas de Driss Chraïbi qui part loin de son pays afin de faire ses études dans un environnement qui lui semble paratopique et où son style littéraire a vu le jour. Chraïbi explique son orientation paratopique dans ces termes : « La langue française, quant à elle, a été un réactif, une distanciation par rapport à mon pays et à moi-même. Cela a élargi mon horizon mais je reste très attaché à mon pays » ${ }^{7}$. Il formule également ce statut de la façon suivante : «Voyageur de deux rives [...] Marocain et Français, moins en termes de territoires géographiques que des territoires imaginaires ». Malgré que ces deux territoires coexistent, ils ne s'influencent pas forcément et créent un tiraillement qui fait de cet entre-deux la condition de l'œuvre du romancier $^{8}$.

Ces déchirements et exactions sont très représentatifs de Driss Chraïbi et de son œuvre littéraire. Elles ont été vécues par une vague d'auteurs qui sont apparus sur la scène littéraire depuis les années 50, vis-à-vis de leur langue et à leur religion. Ainsi, sur le plan personnel, Chraïbi avait coutume dans ses premières œuvres et particulièrement

\footnotetext{
${ }^{4}$ La paratopie désigne le rapport d'appartenance d'un auteur à un milieu, qui rend possibles des énonciations qui excédent l'espace qu'elles ont pour fonction de fonder. In, Dominique Maingueneau, Trouver sa place dans le champ littéraire. Paratopie et création, Louvainla-Neuve, Editions Academia, series: « Au coeur des textes », 2016, p. 187.

${ }^{5}$ Dominique Maingueneau, articles publiés dans des revues, participations à des ouvrages collectifs : Le recours à l'ethos dans l'analyse du discours, Lille, Septentrion, 2004, p. 85.

${ }^{6}$ Idem, p. 74.

${ }^{7}$ Par Driss Chraibi, cit. in ; Ayse Eziler Kiran, L'ethos paratopique d'un narrateur méditerranéen : Le passé simple de Driss Chraïbi, Synergies Turquie, $\mathrm{n}^{\circ}$ 12, 2019, p.52.

${ }^{8}$ Dominique Maingueneau, op., cit. p. 85.
} 
dans Le Passé Simple à montrer un rejet de la composante maghrébine ${ }^{9}$. On assiste alors à un traitement de sujets souvent liés à la révolte contre la soumission familiale et l'aliénation coloniale qui génère l'animosité contre l'occident et la culture de sa propre civilisation, en abordant les aspects les plus importants d'une famille patriarcale et d'une société occidentale sournoise et discriminatoire.

Il s'engage par la suite dans sa littérature dans une démarche de reconquête de son héritage culturel en l'enrichissant d'un goût d'humanisme universaliste, pour enfin entreprendre la modélisation d'un Maghrébin moderniste et vagabond. Cette multitude de facettes dans l'œuvre de Chraïbi aurait accentué son pyrrhonisme et à son esprit décalé un rapport assagi et consolé à la religion et aux anciens traits culturels. Il s'est donc inscrit aux idéaux que l'on octroyait aux Arabes des anciennes civilisations, l'andalouse et la bagdadienne, conçus sur la base d'une délicatesse dans l'amour de la vie et de l'autre à partir de la nourriture, de la poésie et de l'érotique ${ }^{10}$.

Il s'agit donc du processus de l'œuvre littéraire d'un écrivain hors-pair, né au Maroc en 1926 à Mazagan, une petite ville au Maroc à l'époque de la colonisation française. Driss Chraïbi a vécu dans une famille appartenant à la bourgeoisie marocaine de l'époque. Il a commencé son apprentissage dans une école coranique pour ensuite poursuivre ses études dans une école française où il reçoit une éducation à l'occidentale, ceci lui a permis de sortir de sa coquille et de voir le monde extérieur, et c'est ce qui explique en grande partie son tiraillement entre des racines culturelles riches de tradition et une envie insatiable de liberté et de modernité.

Il part ensuite en France où il poursuit ses études supérieures. D'abord en chimie, ensuite dans sa véritable passion qu'est l'écriture, il fait entrer la littérature maghrébine d'expression française dans la modernité.

\footnotetext{
${ }^{9}$ Colette Valat, L'homme du livre de Driss Chraïbi : une utopie pour le prophète, in; Horizons Maghrébins - Le droit à la mémoire, 2007, n 56, p. 81.

${ }^{10}$ Idem, p.83.
} 
En 1954, Chraïbi fait une entrée remarquable en littérature maghrébine francophone avec la publication de sa première œuvre, le Passé simple. Avant de partir à Paris, il avait une représentation idéaliste de l'Occident, pour lui c'était le monde de la modernité. Une fois arrivé en France, il modère la vision qu'il avait de ce pays. " $M a$ vie s'est divisé en deux périodes, 19 ans vécus au Maroc-22 ans en Europe. Et il me semble que dans cette dernière tranche j'ai amassé pas mal d'expériences, dont il faut que je parle. Où est la défection, hein ? Plus tard je reviendrai à la réalité marocaine et arabe ${ }^{11}$.

Cette façon de voir le monde, de modérer sa vision, de comparer les deux cultures, va se traduire dans le roman de Chraïbi. Son œuvre traduira cette soif de la modernité, de la liberté, une soif provoquée par le poids des normes que lui imposait la société dans laquelle il est né et a grandi, une société qui se résumait à l'image de d'une seule figure, celle de son père (I).

En effet, ce roman se caractérise, par rapport aux autres de sa génération, par deux éléments déterminants. D'abord, il se charge manifestement d'une critique sans égal au système de gestion au sein de la famille par une seule personne, le père, un régime patriarcal marocain et qu'il trouve injuste. Ensuite le roman propose un cheminement dans le temps et l'espace de l'intellectuel autochtone distinct de celle que l'on trouve dans la littérature maghrébine d'expression française des années 1950. Un cheminement progressiste et ouvert sur le monde, et sur le changement.

\footnotetext{
${ }^{11}$ Laabi, Abdellatif, Driss Chrä̈bi et nous, défense du passé simple, Souffles, nº5, 2ème année, premier trimestre, 1967, p.08.
} 


\section{I - La figure du père, une critique acharnée du système archaïque marocain.}

Il s'agira d'étudier la force génératrice de ce rebelle contre le pouvoir absolu du père, le seigneur, Driss Chraïbi raconte la vérité sur un pays paralysé par la réduction du statut de l'individu aux traditions de ses ancêtres, sans tenir compte de la liberté individuelle de celui-ci (A). En outre, en pleine bataille pour l'autonomie du pays, il a su non seulement désillusionner le bavardage imposé par les contenus de la littérature antécédente, mais de plus, il s'est montré précurseur dans la dénonciation de toutes les formes d'instrumentalisation des esprits dans un contexte politique et social très tendu $(\mathrm{B})$.

\section{A - LA REDUCTION DU STATUT DE L'HOMME A SES ORIGINES DANS UNE SOCIETE HOLISTE}

Dans les sociétés conservatrices, la personne considérée dans un angle individuel n'a pas d'existence concrète en dehors de sa communauté. Son existence se voit complètement résumée à celle de son groupe ou de sa famille. Sa conduite doit être conforme à celle de la communauté et son autonomie décisionnelle est attachée de manière très conséquente à celle du groupe en question.

La personnalité maghrébine, et la personnalité marocaine notamment, se réduit à celle de la société, de la communauté et de la famille. La place sociale de l'individu est inséparable de la conception d'identité collective ${ }^{12}$.

\footnotetext{
${ }^{12}$ Abdelhak Serhane, L'Amour circoncis, EDDIF, 2002, p. 26.
} 
La personne doit honorer un engagement social dans le cadre duquel les contours de la liberté individuelle sont tracés par le groupe et dont les règles de conduite sont édictées par celui-ci. La personne ou pour plus de précisions, le sujet est complètement dépendant de la communauté à laquelle il est lié par toutes ses actions : la loyauté, le dévouement, le service, voire l'obéissance. Il assure donc une implication dans la communauté puisque celle-ci lui rend la pareille et assure sa protection ou plutôt sa tutelle.

Dans ces sociétés, la personne évolue d'abord au sein de sa famille, ou chaque membre a un statut et un rang prédéfini, et où le père occupe le sommet de la hiérarchie, il incarne l'autorité absolue. Dès son jeune âge, l'individu est exposé à un système patriarcal discrétionnaire. L'enfant est un sujet qui doit le respect et la soumission inconditionnés au père, ce dernier qui représente également le mari et le chef du groupe. Il peut ordonner, interdire ou donner ses directives et même ses ordres en vue de les voir réalisés et mène ce petit groupe dont il est responsable au «droit chemin » et vers voie qu'il estime approprié et juste. La mère ou la femme est également sujette de la même façon aux mêmes règles. Les rapports entre le père et ses sujets sont donc, dans la majorité des situations, des rapports de hiérarchie.

Les individus composant le foyer dont le père est le patron ou seigneur ${ }^{13}$ doivent à leur tour se soumettre aux normes et à la conduite voulues par la communauté dans le sens le plus large. Ce sont ces engagements qui lui garantissent leur place et qui prouvent leur attachement au sein du groupe.

La personne apparaît alors comme un être inconditionnellement lié à son groupe ethnique. Les communautés tribales au Maghreb et en particulier au Maroc à l'époque de la publication du Passé Simple formaient un tout inséparable relié par trois facteurs

\footnotetext{
${ }^{13}$ Terme utilisé dans Le Passé simple de Driss Chraïbi pour désigner le père de Driss dans le Passé Simple, en rapport avec l'emploi de la religion par cette société patriarcale, et ce pour légitimer les abus, non de manière anodine, mais cette expression est utilisée comme figure pour montrer la supériorité du père qui peut aller jusqu'à le considérer comme un être vénérable.
} 
caractéristiques. Il s'agit principalement de la langue commune, de l'aspect religieux (ou culturel lié par définition à la religion) et de la nation. Cette nation, emblème de l'identité du groupe, est guidée par un homme souverain, supérieur à tous, dont la crédibilité et la légitimité sont tirées encore une fois par la langue et la religion, c'est le commandeur des croyants, le seigneur.

Cette représentation caractérisée dans le personnage du père, est une figure et un thème très dominant dans la littérature maghrébine d'expression française, l'essence du rapport de conflit avec cette figure du père prend une place primordiale et joue comme un élément central dans l'œuvre de Driss Chraïbi. Il s'agit pour lui d'un personnage central qui détient toute l'attention, prend tous les angles de la scène dans la société marocaine réduisant les enfants et la mère en un rien, des personnages fictifs qui vivent sous son ombre. Le père est un objet d'un discours agressif, le sujet du père reste un sujet très sensible.

Les rapports entre le père et les autres membres de sa famille, ses enfants, sa femme, sont des rapports de domination et de supériorité. Le père est le plus apte, physiquement et mentalement, car c'est à lui que reviennent toutes les décisions importantes, c'est lui qui peut donner des ordres, qui s'occupe de la gestion. La prise de position ou l'affirmation de soi chez l'enfant ou la femme/mère (puisque tous deux sont assimilés dans le même rôle) ne peuvent avoir lieu dans la structure traditionnelle marocaine tant que le père est présent ${ }^{14}$.

L'exemple du père Ferdi illustre ce genre de personnage, appelé le seigneur, figure emblématique de l'homme fort dans la société marocaine. Il est de la nature de ceux qui forcent le respect de leur entourage, qui cachent par leur existence celle des autres, cela se comprend encore plus que sa richesse et sa noblesse lui procurent plus de légitimité et de prestige dans sa communauté. Par conséquent, il joue bien ce rôle, il ne se montre d'aucune considération pour sa femme ou ses enfants et ne permet pas que ses ordres soient contestés ou débattus.

\footnotetext{
${ }^{14}$ Abdelhak Serhane, L'Amour circoncis, op. cit., p. 56.
} 
Cette appropriation de la rébellion du jeune Driss Ferdi avait toute sa place et son importance dans le contexte dans lequel le Passé simple est édité. Driss Chraïbi a été parmi les premiers auteurs qui ont osé prendre les devants afin de dénoncer certaines formes d'injustices dans la société marocaine. L'œuvre justifiait à elle seule en quelque sorte la présence libératrice du colonialisme à l'époque des années cinquante.

\section{B - CHRAÏBI, INITIATEUR DE LA REVOLTE CONTRE LES FORMES DE PATRIARCAT DANS UN CONTEXTE SOCIAL TENDU.}

Le rôle du père au sein du noyau familial s'analyse de différents angles, d'abord historique, ensuite social, et finalement d'un angle culturel. «En occident la figure symbolique du père, celle qui imprègne les consciences fondamentales et principales, notre imaginaire collectif depuis la préhistorique jusqu'à nos jours, repose sur deux idées fortes : la première, à caractère juridique, rassemble les prérogatives de la puissance paternelle et cordonne les éléments de patriarcat ${ }^{15}$.

Dans le contexte maghrébin en revanche, les individus composant le noyau familial sont extrêmement incorporés. Le père est en même temps le maitre ou le patron. Dans sa relation avec sa femme, il n'est pas exclusivement lié par des liens affectifs, ils sont plutôt liés par une relation de collaboration dans une tâche dont l'intérêt est commun. Les enfants contribuent, en même que les parents, au même processus restreint de division du travail. L'entité familiale leur inculque non seulement les principes de base de la socialisation, mais leur procure également les connaissances spécifiques dont ils ont besoin, assure l'instruction...Le lien est important et indissociable entre le père et

\footnotetext{
${ }^{15}$ J-M. Pétillon, C. Darmangeat, Histoire et préhistoire de la domination masculine, Hal Archives ouvertes, 2018, p.153.
} 
les membres de la famille parce que son autorité ne cesse jamais de s'exercer sur ceuxci. Autrement, il ne cesse jamais d'être le patron, il est constamment en le guide et le maitre entendu. La famille forme ainsi un vrai clan où le père est le chef incontesté.

Ce dernier enjoint sa famille, par sa situation de puissance, à le respecter et à se soumettre à ses ordres. Habituellement, les enfants ainsi que la femme ne peuvent pas désavouer cet agencement de la famille que forme la supériorité du père. Cependant, les choses ne se passent pas toujours ainsi. Il est possible que le pouvoir du père soit contredit et mis en exergue par les enfants ou par la femme.

Ce qui fait la particularité du l'œuvre de Chraïbi, que l'on peut qualifier de semiautobiographique, et de l'autobiographie marocaine de manière générale. C'est principalement, ce refus de se soumettre à cet ordre instauré et justifié par une coutume tribale séculaire.

On constate alors dans le Passé Simple que la relation entre le Seigneur, père de la famille Fardi, et son fils Driss, personnage principal du roman, est souvent problématique voire conflictuelle.

Ces rapports antagoniques entre père et fils sont illustrés souvent de la même manière à travers les ouvrages littéraires marocains d'expression française. La subordination des enfants, imposée par le père, est souvent sujette à des formes de rébellion et de violence. La figure du père dans ces œuvres, surtout autobiographiques, est souvent décrite de manière péjorative. Karim Nasseri, par exemple, au même titre, désigne son père «le dictateur», un surnom qu'on retrouve tout au long de ses deux textes autobiographiques. Dans sa première œuvre, intitulée «Chroniques d'un enfant du hammam », le narrateur parle de son père : «J'entendis le Dictateur m'appeler aux travaux forcés ${ }^{16}$

\footnotetext{
${ }^{16}$ Karim Nasseri, Chronique d'un enfant du hammam, Paris, Denoêl, 1998, p. 13.
} 
Dans le roman Le Passé Simple, objet de notre étude, le pouvoir est résumé dans la seule personne qui est le père, qu'il nomme à son tour, le seigneur. Cette relation père/fils est alors traitée par Chraïbi sous différents angles, parfois choquants dans le contexte de l'époque.

En effet, de par son double bagage culturel, et suite à son instruction reçue à l'école française durant la colonisation française au Maroc, et l'éducation arabo-musulmane qu'il a reçue au sein de sa famille, tous ces facteurs ont été des éléments décisifs dans l'œuvre de Driss Chraïbi et dans le Passé simple particulièrement. Cet enseignement lui a permis de mettre sa propre personne en avant, et de mettre en cause la figure du père, personne sacralisée dans la société et dans la culture marocaine.

C'est donc pour la première fois, Chraïbi, novateur de cette littérature, particulièrement dans ce contexte, provoqua un énorme vacarme dans la société marocaine. Notre novateur y désillusionne les genres de littératures antécédentes. Il a en effet dénoncé toutes ces pratiques injustes et inviolables de sa société. Du côté du colonisateur, cette œuvre fut une conjoncture avantageuse, il s'agissait pour eux d'un écrivain local qui saisissait la plume pour dénoncer les outrages de la société et du régime politique de son pays, complice dans l'instauration de ces pratiques, désavouer sa complicité et accorder une certaine légitimité au Protectorat. En Europe, cet ouvrage fut reçu à l'instar d'un véritable coup de couteau, porté essentiellement aux indépendantistes, qui luttaient promptement pour la liberté de leur pays.

Cette œuvre, rédigé à la première personne du singulier, met en œuvre un jeune homme, Driss Ferdi, qui se rebelle contre son père et contre la société marocaine. Il menace alors les fondements culturels et traditions séculaires de celle-ci, aux enseignements religieux puisqu'il s'attaquait à la pratique hypocrite de la religion qui n'en fait pas réellement partie. Au discours complaisant d'antan vint se substituer une accusation violente des maux affectant la société Marocaine et des tabous existant dans celle-ci. 
Les mots se transforment ainsi en une arme très puissante, destinés à brosser la vérité telle qu'elle est et non plus à manipuler les esprits par un système de despotisme. Il s'agit pour lui d'en finir une fois pour toutes avec cette langue de bois, qui prohibe aux individus de prendre leur destin en main, de parler de certains tabous, ou qui les pousse à les dire d'une telle manière que ça rentre dans les critères et selon les conditions du bon vouloir d'une catégorie d'individus de la société marocaine, qui impose sa vision du monde.

Notre auteur, par la pluralité des langages et des cultures, et à travers sa parodie des textes religieux et culturels instrumentalisés, créa une langue transgressive, sauveteuse, réduisant en miette cette langue de bois, Driss Chraïbi dans son œuvre. Il donne en outre à son personnage Driss Ferdi l'aptitude afin de lutter contre sa condition et dans sa relation avec son père.

C'est cette éducation européenne qui a permis à Driss Ferdi de percevoir que ce ne sont pas les espaces géographiques qui partagent son peuple de celui qui se trouve sur l'autre rive de la méditerranée, mais ce sont plutôt des espaces d'une nature différente. L'écrivain, en s'accoutumant avec la culture occidentale en étant présent sur sa terre natale, et où la situation historique lui impose les valeurs occidentales, il se retrouve exposé à deux mondes différents. "Dédaigneux des protocoles, parce que mes vêtements sont européens et que je suis presque européanisé $»{ }^{17}$. Le jeune Ferdi ne trouve plus place dans la société marocaine. Par sa révolte, il veut s'affirmer et renier le Maghreb dont il est issu.

Cette question d'identité, occupe principalement le jeune homme, notion complexe et omniprésente dans l'environnement de la littérature mondiale fréquemment et la littérature maghrébine d'expression française en particulier, ce tiraillement identitaire qui ne cesse de faire figure. Nous ne pouvons parler du Passé simple sans réfléchir au problème identitaire du Maroc et des marocains, surtout ceux d'expression française,

\footnotetext{
${ }^{17}$ Chraïbi, Driss, Le passé simple, Ed, Denoël, « folio », Paris, 1954.P.16
} 
que nous pouvons justifier par le volet historique, plus exactement par le facteur de la colonisation qui a fait apparaitre un choc culturel et de civilisations.

\section{II - Le paradoxe d'un tiraillement entre héritage ancestrale et modernisme occidental.}

Dans le Passé Simple, Driss Ferdi, le personnage principal du roman de Chraïbi, renverse un grand nombre de systèmes de valeurs dans la société marocaine, comme les coutumes familiales et les modèles vertueux commandés par la religion. Driss Ferdi représente en quelque sorte le désarroi de l'auteur du livre (B), mais fait partie également et plus globalement de cette nouvelle génération qui est trainée entre deux cultures, celle de sa terre natale (le fardeau de la tradition, religion considérée comme un instrument et détourner pour servir des fins immorales et le système archaïque et patriarcal), et celle que l'occident tente d'inculquer à ce pays (A). Driss doit trouver sa place entre les deux.

\section{A - LE ROLE DU COLONIALISME DANS LA CREATION D'UNE MENTALITE DECHIREE.}

Le Passé Simple est l'œuvre semi-autobiographique d'un jeune Marocain, Driss, représentant indirectement l'auteur de l'œuvre, qui vivait dans la ville de Fès. Appartenant à une famille de la bourgeoisie marocaine de l'époque, Driss le personnage principal, vit dans une situation d'ambiguïté et d'inconstance. Agité entre deux cultures différentes, la culture arabo-musulmane et la culture française, causée par le protectorat imposé au Maroc par la France, pays colonisateur. Driss se voit très 
rapidement devenir victime d'un ébranlement d'identité généré par la place qu'il occupe entre ces deux systèmes symboliques et antinomiques.

Pour poursuivre son pouvoir hérité de ses ancêtres depuis des siècles, le « Seigneur », le chef de la famille et père de Driss, tient à façonner la mentalité du protagoniste. En effet, La France, considérée par le père dénommé le Seigneur dans le Passé Simple, comme un danger, un ennemi et surtout comme un espace néfaste. Les extraits suivants se portent garantes de ces dominantes de signification : «nous sommes au siècle des califes, tu seras du siècle vingt. Nous t'introduisons dans le camp ennemi afin que tu te familiarises avec ses armes. Cela et pas autre chose ${ }^{18}$ s'acharne-t-il à inculquer à son fils. Mais cet Occident va être perçu par Driss comme un lieu où règne l'égalité, la liberté et la justice. Le colonialisme va offrir à Driss le scepticisme indispensable qu'il lui permettra de rejeter sa propre culture et s'affermir contre le pouvoir despotique et théocratique de son père. Le «Seigneur ». Les nouvelles valeurs culturelles qui lui sont prescrites à l'école ont trouvé une oreille attentive et ont vite converti celles de la famille et l'ont aidé à se délivrer momentanément de sa raideur.

Dans cette situation paradoxale, l'enseignement français qu'il a reçu à l'école française a donné à Driss tout le courage de réagir brutalement contre son père. Cette face théocratique et arbitraire qui est la représentation du passé dans toutes ses facettes de contrainte et d'exclusion. Driss rejette donc cet ordre et ses constituantes théoriques. La fausseté du système de valeurs coloniales avait, bien sûr, incité Driss à admettre l'hypocrisie, l'injustice et la corruption de sa propre société.

Le Passé simple écrit à l'aube de l'indépendance du Maroc a été alors le produit des années de colonialisme que le pays a dû subir. À cette époque-là, la population marocaine était sous un système de protectorat français, et l'auteur, comme choqué par les deux cultures reçues, toutes opposées, commence alors une quête de recherche de soi, pose une multitude de questions, se révolte contre les deux systèmes, commençant

\footnotetext{
${ }^{18}$ Chraïbi, Driss, Le passé simple, op. cit., p. 188-189.
} 
par celui-ci dont l'injustice parait la plus évidente, celui de son pays natal. Il commence à s'interroger, comme le fait tout le peuple marocain, comment devrait vivre l'avenir du Maroc, sous l'influence du modernisme qu'a instauré la France ou comme un pays totalement indépendant qui conserve sa propre culture et ses propres valeurs.

Le Passé simple était la première œuvre à relater ces questions, à s'interroger sur e cette division entre le pays des origines et la nouveauté de la modernité occidentale. Dans ces dernières années de l'occupation coloniale, chaque écrit nouveau fut scruté de tout près, aussi bien par les occidentaux que par les autochtones. Les européens étaient curieux de savoir ce qui se passait dans la société marocaine et ces derniers voulaient connaitre quel écho produirait cette œuvre.

L'originalité de l'auteur consiste par ailleurs en sa capacité à ébranler les fondements de deux discours accaparant l'espace rationnel : le discours colonialiste avec toutes ses expressions orientalistes, et d'un autre coté le discours marocain et son archaïsme séculaire désuet. Il tente aussi d'élucider la question autour des fondements du discours égocentrique occidental en se déplaçant par rapport à ses fondements littéraires artistiques et culturels. Le projet de l'auteur se résume donc dans la remise en question du bien-fondé de tout discours autoritaire et à ouvrir l'horizon de l'épanouissement aux êtres désocialisés, à ceux qui ne font partie ni du monde du Seigneur, ni de celui du colonisateur.

\section{B - LE DESARROI D'UN AUTEUR REVOLTE NOSTALGIQUE DE LA \\ CULTURE DE SA TERRE NATALE.}

Le Passé Simple occupe une place singulière dans le corpus littéraire maghrébin d'expression française. Euvre controversée depuis sa première publication, elle 
continue d'être considérée comme le livre le plus controverse de la génération des années cinquante.

Il est considéré comme un texte prémonitoire de la littérature de la décolonisation des années soixante et soixante-dix par son caractère rebelle et fondateur, Le Passé Simple est un espace littéral où coexistent à la fois l'archaïque et le post-moderne. Sa trajectoire narrative se construit selon un mouvement de va-et-vient continu entre le passé et le présent. Un passé contesté, vu sa mesure constant et immobile, et un présent fractionné, constitué de facteurs disparates qui ne peuvent avoir une valeur que ajustés dans leur contexte de référence.

A la différence d'autres récits qui se transcrivent dans la tradition ethnographique, Le Passé Simple profite d'un statut distinctif en ce qui concerne le parcours de son auteur. Il illustre en effet le désarroi de celui-ci. Il est marqué par le déploiement d'une philosophie caractérisée par une violence textuelle, qui se distingue par le biais d'une composition narrative sectorielle et une écriture qui fusionne un assemblage d'un vocabulaire brassé de la tradition littéraire française et un vocabulaire résultant d'une nostalgie de la culture orale marocaine.

Ceci est illustré dans le personnage principal de son roman, Driss qui, incapable de se construire une nouvelle identité, reste piégé entre deux mondes différents, voire conflictuels : l'oriental, abandonné en raison de son archaïsme et l'occidental, qui ne soutienne aucune diversité. Les nouvelles valeurs que le colonisateur lui a inculquées lui ont permis d'ouvrir les yeux sur une réalité amère. D'abord, celle des pratiques d'une société sans scrupule conçue sur le mensonge, les mythes d'une religion malheureusement mal comprise et instrumentalisée, dépourvue par les hommes de sa spiritualité, une société construite par et pour les hommes pour assumer la volonté divine par le père "le seigneur » Le passé simple constitue comme une « littérature de scandale qui vise à démasquer le refoulé social et politique des idées et des langages 
reçus, à déplacer les censures qui le cachent » ${ }^{19}$. D'un autre côté, Driss Chraïbi, illustre dans le Passé Simple les manifestations de l'hypocrisie de son père comme celles de l'hypocrisie de colonisateur, les deux protagonistes sont comparables dans cette figure. Il se sent trahi et a de plus en plus le sentiment de s'être bercé d'illusions. Il prend terriblement conscience que l'Occident est en train de lui fermer sa porte, progressivement. Il ne sait plus où se diriger et se sent défavorisé : Je viens de m'engager dans votre route messieurs. Je ne m'y suis engagé vierge, mais tel dans le mariage un conjoint « ayant beaucoup souffert ». En conséquence, si je dis : "Liberté, Égalité, Fraternité : devise aussi rouillée que la nôtre », vous me comprenez $»^{20}$.

Driss va donc vouloir se battre pour jouir pleinement et gagner son indépendance, Le jeune Ferdi sait que l'accueil va être très difficile car dès que le seigneur aura fait l'autochtone à son image, et son père, affermi par ces propres valeurs individuels, qu'il va s'imposer et revendiquer une place dans ce monde sans considération des fils, ce qui est tout à fait normal pour lui ; Driss, ayant fréquenté l'école française, a adopté les grandes valeurs de Droits de l'homme, Liberté, Égalité, mais dans la vie de tous les jours, tout cela semble désuet et utopique.

Au nom de ces nouvelles convictions, il s'est rebellé contre le pouvoir autoritaire de son père, l'oppression familiale, la société musulmane, mais l'Occident lui refuse tout autant de lui accorder une place. Voici venu pour lui le temps des désenchantements car la culture occidentale n'est pas aussi extraordinaire ni parfaite qu'elle n'y paraît. Pourtant, à cause du colonialisme d'abord, et de son père ensuite, il décide de partir à l'autre rive de la méditerranée, en ayant la conviction de réussir et de gagner sa liberté totale.

Driss Ferdi représente Driss Chraïbi dans le Passé Simple dans la mesure où il est partagé entre le penchant aux valeurs ancestrales et l'attrait de la modernité. Le jeune

\footnotetext{
${ }^{19}$ Mitterrand. Henri, Coup de pistolet dans un concert : Une saison dans la vie d'Emmanuel, voix et images, in ; Presses de l'Université du Québec, Volume 2, N³, 1977, p. 414

${ }^{20}$ CHRAIBI, Driss, Le Passé simple, Op.cit, p.163.
} 
Driss se cherche, il est tiraillé entre les injustices du système colonisateur et celles du régime patriarcal marocain : «Certes Occident je me scinde, mais mon identité est une infinité de jeux, de roses de sable, euphorbe est ma mère, désert est ma mère, je suis projeté Occident. ${ }^{21}$.

\section{Conclusion.}

Driss Chraïbi l'un des grands auteurs marocains d'expression, fait partie de cette génération qui est née à l'aube de l'indépendance du Maroc, à travers son Passé simple à tendance semi autobiographique, il était précurseur de cette vague d'écrivains maghrébins révoltés. C'est grâce également à son œuvre originale sortie en 1954, qu'il était considéré comme le seul auteur à écrire une littérature avec la langue du colon avec un style révolutionnaire.

L'œuvre de Chraïbi a en effet fait raisonner à l'image d'une bombe dans la société marocaine, mais a secoué en même temps les esprits de l'occident. Il s'agit en effet d'un écrivain hors norme, un écrivain complétement atypique, par son style révolutionnaire, qui pointe du doigt les défaillances du système tout en s'abstenant de donner une vision générale de la société marocaine et du système patriarcal ancestral et soumis qui ordonne la société marocaine.

D'une modernité inouïe, Le passé simple est une combine de la tradition littéraire française avec un fonds culturel maghrébin. Ainsi, il montre à travers son personnage, Driss Ferdi, qui représente en quelque sorte son parcours sociétal au sein de la société marocaine, que nonobstant sa fascination pour l'Europe et ses idéaux, Ferdi reste néanmoins toujours attaché à ses origines et à la culture de son pays natal. Par

\footnotetext{
${ }^{21}$ Abdelkabir. Khatibi, La mémoire tatouée, Denoël, Paris, 1971, p.173.
} 
conséquent, il existe certains éléments qui ne lui permettent pas d'adhérer entièrement à cette culture à la française dite moderne.

La culture où Driss a vécue et a grandi a marqué son être, cela lui occasionne un désarroi identitaire, son identité est en malaise, il est tiraillé entre l'influence des deux cultures, toutes les deux imparfaites. Le personnage de Chraïbi a essayé de réaliser sa submersion poétique à travers son œuvre, une descente aux enfers pour trouver son être, trouver la liberté et la modernité auxquelles il aspirait, et avoir la possibilité de mettre à nu tous les abus qui foisonnaient au sein sa société.

Or, cette expérience de recherche d'autonomie et sa détermination de se libérer de l'archaïsme de sa société, ainsi que toutes ces valeurs qui se manifestent dans son milieu d'origine est une fabrique à mi-chemin, car le personnage chraïbien ne parvient pas à se séparer entièrement ni de ce milieu ni des siens. Il est tiraillé entre deux cultures complètement à l'opposé, cela fait de lui une proie d'un malaise identitaire généré par le parallèle de deux fondements culturels radicalement différents : «Tous les problèmes et crises de l'identité sont dus à une quelconque frustration ou atteinte à un ou plusieurs de ses sentiments $»^{22}$

En revanche, à la différence de Driss Chraïbi et de son style littéraire, les nouveaux écrivains d'expression française, tel qu'Abdellah Baida ou Maria Guessous, défendent plutôt un langage rassérénée dans leurs écrits et ne paraissent pas faire de reproduction du style révolté de Driss Chraïbi ni d'aucun autre auteur notable de la littérature maghrébine d'expression française (Rachid Boudjedra, Rachid Mimouni, Tahar Ben Jelloun, ...) ni sur le point de vue thématique ni sur le plan esthétique. Ceci étant, Chraïbi a ouvert la voie à travers son œuvre à une création libre qui marque un point de séparation avec une littérature sourde et complaisante.

\footnotetext{
${ }^{22}$ Mucchielli. Alex, Problèmes et crises de l'identité psychosociale, in ; L'identité (2013), p. 95.
} 


\section{Bibliographie.}

AIT AMEUR. Thinhinene, BOUAMRA Mebrouka, La quête identitaire dans Le passé simple de Driss Chraïbi, Université Mouloud Mammeri de Tizi-Ouzou, 2017.

ARON, Paul, SAINT-JACQUES, Denis, VIALA, Alain, le dictionnaire de littérature, Paris, PUF, 2002.

Belkaid. Amaria, L'Expression contestataire chez Driss Chraïbi, Synergies Algérie n ${ }^{\circ}$ $13,2011$.

Bouazza.Abdelhak, Le passé simple de Chraïbi, soubassements d'une révolte précoce, Revue Annales du patrimoine, Université de Mostaganem, n² 20, 2020.

Chraïbi. Driss, Le passé simple, Ed, Denoël, « folio », Paris, 1954.

Déjeux. Jean, L'étrangère dans la littérature maghrébine de langue française, in ; Hommes \& Migrations, 1993. 
Déjeux. Jean, Littérature maghrébine de langue française, Editions Naaman, Québec, 1980.

El Bouziki. Mohammed, L'Orient et l'Occident : étude sémantique et interculturelle, Publications du Centre Universitaire de Langues et Communication, Université Mohammed Premier, Oujda, 2017.

Eziler Kiran. Ayse, L'ethos paratopique d'un narrateur méditerranéen : Le passé simple de Driss Chrä̈bi, Synergies Turquie, $\mathrm{n}^{\circ}$ 12, 2019.

Hirchi. Mohammed, La réception du Passé Simple de Driss Chrä̈bi en 1954, in ; Etudes Francophones, Vol. 26, $\mathrm{n}^{\circ} 1$ et 2, Colorado State University.

Kadra-Hadjadji. Houaria, Contestation et révolte dans l'œuvre de Driss Chrä̈bi, Publisud, Mimizan, 1986.

Khatibi. Abdelkabir, La mémoire tatouée, Denoël, Paris, 1971.

Laâbi, Abdellatif, Driss Chraïbi et nous, défense du passé simple, Souffles, $\mathrm{n}^{\circ} 05$, 2ème année, premier trimestre, 1967.

Laâbi, Abdellatif : Droits de l'Homme et littérature engagée au Maroc, in Horizons Maghrébins, $n^{\circ} 11,1987$.

Luica. Larissa-Daiana, Écriture autobiographique et pseudo-autobiographique dans l'oeuvre de Driss Chraïbi, Thèse de doctorat, Université Bordeaux 3, 2013.

Maingueneau. Dominique, articles publiés dans des revues, participations à des ouvrages collectifs: Le recours à l'ethos dans l'analyse $d u$ discours, Lille, Septentrion, 2004. 
Maingueneau. Dominique, Trouver sa place dans le champ littéraire. Paratopie et création, Louvain-la-Neuve, Editions Academia, series: « Au coeur des textes », 2016.

Mitterrand. Henri, Coup de pistolet dans un concert : Une saison dans la vie d'Emmanuel, voix et images, in ; Presses de l'Université du Québec, Volume 2, $\mathrm{N}^{\circ} 3$, 1977.

Mucchielli. Alex, Problèmes et crises de l'identité psychosociale, in ; L'identité, 2013.

Nasseri. Karim, Chronique d'un enfant du hammam, Paris, Denoêl, 1998.

Pétillon. J-M., Darmangeat. C., Histoire et préhistoire de la domination masculine, Hal Archives ouvertes, 2018.

Saad Aroussi, La figure $d u$ père dans Le passé simple de Driss Chraïbi, Mémoire pour l'obtention d'un master, Université Msila, 2019.

Serhane. Abdelhak, L'Amour circoncis, EDDIF, 2002.

Triaud. Jean-Louis, Une laïcité coloniale. L'administration française et l'islam en Afrique de l'ouest, Politique, religion et laïcité, P. U. de Provence, Aix-en-Provence 2009.

Valat. Colette, L'homme du livre de Driss Chraïbi : une utopie pour le prophète, in ; Horizons Maghrébins - Le droit à la mémoire, nº 56, 2007.

Yetiv. Isaac, L'aliénation dans le roman maghrébin contemporain, in; Revue des mondes musulmans et de la Méditerranée, n $^{\circ} 18,1974$. 